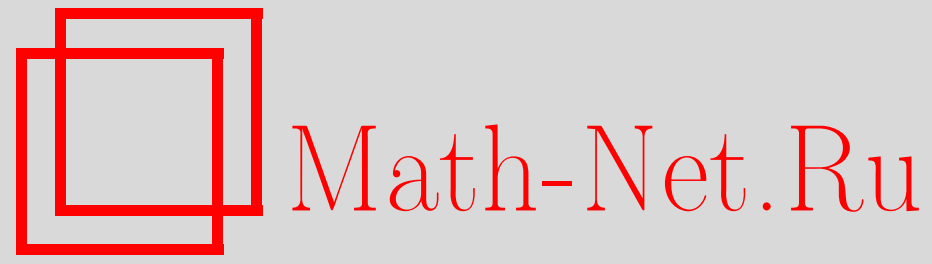

Общероссийский математический портал

И. Л. Блошанский, О. К. Иванова, Т. Ю. Рослова, Обобщенная локализация и равносходимость разложений в двойной тригонометрический ряд и интеграл Фурье функций из $L\left(\ln ^{+} L\right)^{2}$, Матем. заметки, 1996, том 60, выпуск 3, 437-441

DOI: https://doi.org/10.4213/mzm1844 
Использование Общероссийского математического портала MathNet.Ru подразумевает, что вы прочитали и согласны с пользовательским соглашением

http://www . mathnet.ru/rus/agreement

Параметры загрузки:

IP : 54.89 .56 .158

26 апреля 2023 г., 14:54:12

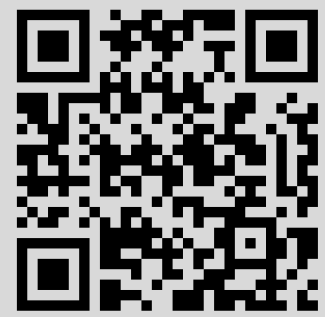




\section{ОБОБЩЕННАЯ ЛОКАЛИЗАЦИЯ И РАВНОСХОДИМОСТЬ РАЗЛОЖЕНИЙ В ДВОЙНОЙ ТРИГОНОМЕТРИЧЕСКИЙ РЯД И ИНТЕГРАЛ ФУРЬЕ ФУНКЦИЙ ИЗ $L\left(\ln ^{+} L\right)^{2}$}

\section{И. Л. Блошанский, О.К. Иванова, Т. Ю. Рослова}

1. Рассмотрим $N$-мерное евклидово пространство, элементы которого будем обозначать $x=\left(x_{1}, \ldots, x_{N}\right)$, и положим $x z=x_{1} z_{1}+\cdots+x_{N} z_{N}$.

Для любого $\lambda \in \mathbb{R}^{1}$ определим множество

$$
\mathbb{R}_{\lambda}^{N}=\left\{x \in \mathbb{R}^{N}: x_{j} \geqslant \lambda, j=1, \ldots, N\right\} .
$$

Введем также множество $\mathbb{Z}^{N} \subset \mathbb{R}^{N}$ всех векторов с целочисленными координатами и положим $\mathbb{Z}_{\lambda}^{N}=\mathbb{Z}^{N} \cap \mathbb{R}_{\lambda}^{N}$.

Пусть $\Phi:[0, \infty) \rightarrow[0, \infty)$ - неубывающая функция, через $\Phi(L)=\Phi(L)\left(T^{N}\right)$ обозначим множество суммируемых функций $f$ таких, что

$$
\|f\|_{\Phi(L)}=\int_{T^{N}} \Phi(|f(x)|) d x<\infty
$$

где $T^{N}=\left\{x \in \mathbb{R}^{N}:-\pi \leqslant x_{j}<\pi, j=1, \ldots, N\right\}$. Если $\Phi(u)=u^{p}$, то обозначим $\Phi(L)=L_{p}\left(T^{N}\right), p \geqslant 1$, если $\Phi(u)=u \ln ^{+} u$, где $\ln ^{+} u=\max \{1, u\}$, то $\Phi(L)=L \ln ^{+} L$.

Работа вьполнена при финансовой поддержке Российского фонда фундаментальных исследований, грант № 96-01-00332. Кроме того, работа первого автора выполнена также при поддержке Государственного Комитета Р $\Phi$ по делам науки и высшей школы, грант № 94-1.2-71(26).

(С) И. Л. БЛОШАНСКИЙ, О.К. ИвАНОВА, Т. Ю. РОСЛОВА 
Пусть $2 \pi$-периодическая функция $f(x) \in \Phi(L)\left(T^{N}\right), N \geqslant 1$, разложена в кратный тригонометрический ряд Фурье:

$$
f(x) \sim \sum_{k \in \mathbb{Z}^{N}} c_{k} e^{i k x}
$$

Для любого вектора $n=\left(n_{1}, \ldots, n_{N}\right) \in \mathbb{Z}_{1}^{N}$ рассмотрим прямоугольную частичную сумму

$$
S_{n}(x ; f)=\sum_{\left|k_{1}\right| \leqslant n_{1}} \cdots \sum_{\left|k_{N}\right| \leqslant n_{N}} e^{i\left(k_{1} x_{1}+\cdots+k_{N} x_{N}\right)},
$$

частным случаем которой является квадратная частичная сумма $S_{n_{0}}(x ; f)$, когда $n_{1}=n_{2}=\cdots=n_{N}=n_{0}$.

При этом под сходимостью ряда в (1) по прямоугольникам будем понимать существование предела частичных сумм $S_{n}(x ; f)(2)$ при $n \rightarrow \infty$ (иными словами, $\left.\min _{1 \leqslant j \leqslant N} n_{j} \rightarrow \infty\right)$, а под сходимостью ряда в (1) по квадратам - существование предела $S_{n_{0}}(x ; f)$ при $n_{0} \rightarrow \infty$.

Пусть $\Omega$ - произвольное измеримое множество, $\Omega \subset T^{N}, \mu \Omega>0\left(\mu=\mu_{N}-\right.$ $N$-мерная мера Лебега), и пусть $f(x)=0$ на $\Omega$.

2. Отсутствие классической локализации для тригонометрического ряда Фурье в классе $L_{p}, p \geqslant 1$, в кратном случае привело одного из авторов к понятию обобщенная локализация почти всюду (п.в.) [1] (см. также [2]).

ОПРЕДЕЛЕНИЕ. Пусть $\Omega, \Omega \subset T^{N},-$ произвольное множество положительной меры. Будем говорить, что для кратных рядов Фурье функций из класса $\Phi(L)$ справедлива на множестве $\Omega$ обобщенная локализация почти всюду (ОЛ), если из условия $f \in \Phi(L)\left(T^{N}\right), f(x)=0$ на $\Omega$, следует, что почти всюду на $\Omega$ существует предел

$$
\lim _{n \rightarrow \infty} S_{n}(x ; f)=0 .
$$

При $N=1$ ОЛ справедлива на любых измеримых множествах $\Omega \subset T^{1}$ в классах $L_{p}, p>1$. Это следует из работ Л. Карлесона [3] и Р. Ханта [4]. Если $p=1$, то ОЛ в одномерном случае справедлива в классе $L_{1}$ на измеримом множестве $\Omega \subset T^{1}$, $\mu \Omega>0$, тогда и тольо тогда, когда это множество является открытым п.в.

Достаточность этого факта следует из классического принципа локализации Римана, необходимость доказана И. Л. Блошанским (см. [5, теорема 2 для $N=1]$ ). (Множество $E$ будем назьвать (см. [5]) открытым почти всюду, если существует открытое множество $E_{1}$ такое, что $\mu\left(E \Delta E_{1}\right)=0$.) В кратном случае исследования ОЛ были также проведены И. Л. Блошанским в работах [1], [2], [5]-[7]. Так в случае $N=2$ в работе [2] был доказан обобщенный принцип локализации п.в., заключающийся в том, что для функций $f \in L_{p}\left(T^{N}\right), p>1$, сходимость или расходимость двойного ряда Фурье (функции $f$ ) п.в. в некотором круге не зависит от поведения $f$ вне этого круга. (В силу результатов по равносходимости разложений в двойной ряд и интеграл Фурье функций из $L_{p}, p>1$, (см. [2], а также обзор, данный ниже) указанный принцип верен и для двойных интегралов Фурье.) Таким образом, для двойных рядов Фурье (суммируемых по прямоугольникам) на открытых п.в. множествах ОЛ справедлива в классах $L_{p}, p>1$. 
Заметим, что усилить последний результат, доказав его в случае $N=2, p>1$ для произвольного измеримого множества, оказалось невозможньм, так как в работе [6] было построено измеримое множество $\Omega \subset T^{2}$ (с мерой, сколь угодно мало отличающейся от меры квадрата $T^{2}$ ), на котором ОЛ не справедлива в классе $L_{\infty}\left(T^{2}\right)$ (при суммировании по прямоугольникам).

Что касается $N \geqslant 3$, то (см. [7]) ОЛ в этом случае уже не справедлива ни на каком измеримом множестве $\Omega \subset T^{N}$, не являющемся плотным в $T^{N},\left(\bar{\Omega} \nsupseteq T^{N}, \bar{\Omega}\right.$ замыкание множества $\Omega$ ), при суммировании кратного ряда Фурье по прямоугольникам даже в классе $\mathbb{C}\left(T^{N}\right)$.

Если же рассматривать класс $L_{1}\left(T^{N}\right)$, то (см. [5]), уже начиная с двумерного случая, ОЛ не справедлива вообще ни на каком измеримом множестве $\Omega \subset T^{N}$, $0<\mu \Omega<(2 \pi)^{N}, N \geqslant 2$, даже при суммировании кратного ряда Фурье по квадратам.

Сформулированные выше резуль таты естественно ставят вопрос о справедливости ОЛ (на открытых множествах) в классах $\Phi(L)\left(T^{N}\right)$ при $N=2$.

3. Если $f \in L\left(\ln ^{+} L\right)^{2} \ln ^{+} \ln ^{+} L\left(T^{2}\right)$, то как следует из работы П. Шёлина [8], ряд Фурье функции $f$ сходится п.в. на $T^{2}$ при суммировании по квадратам. В случае суммирования по прямоугольникам, как известно [9], двойной ряд Фурье функции $f$ может неограниченно расходиться п.в. на $T^{2}$, даже если $f \in \mathbb{C}\left(T^{2}\right)$.

Таким образом, в классе $L\left(\ln ^{+} L\right)^{2} \ln ^{+} \ln ^{+} L\left(T^{2}\right)$ ОЛ для двойных рядов Фурье справедлива на любых измеримых множествах (в случае суммирования по квадратам). При $N=1$ ОЛ справедлива на любых измеримых множествах в классе $L \ln ^{+} L \ln ^{+} \ln ^{+} L$ (это следует из той же работы П. Шёлина [8]).

4. В настоящей работе нами получен следующий результат, касающийся ОЛ в классе $L\left(\ln ^{+} L\right)^{2}\left(T^{2}\right)$ при суммировании двойного ряда Фурье по прямоугольникам.

Теорема 1. Пусть $\Omega$ - произвольное (непустое) открытое мнохсество $\Omega \subset T^{2}$, и пусть $f \in L\left(\ln ^{+} L\right)^{2}\left(T^{2}\right), f(x)=0$ на $\Omega$. Тогда

$$
\lim _{n \rightarrow \infty} S_{n}(x ; f)=0 \quad \text { для почти всех } x \in \Omega \text {. }
$$

Более того, для любого $\varepsilon>0$ существует открытое мнохсество $\Omega_{\varepsilon} \subset \Omega$, $\mu \Omega_{\varepsilon}>\mu \Omega-\varepsilon$, иостоянная $C_{\varepsilon}>0$ такие, что

$$
\left\|\sup _{n \in \mathbb{Z}_{0}^{2}}\left|S_{n}(x ; f)\right|\right\|_{L_{1}\left(\Omega_{\varepsilon}\right)} \leqslant C_{\varepsilon} \int_{T^{2}}|f(x)|\left(\ln ^{+}|f(x)|\right)^{2} d x+C_{\varepsilon} .
$$

Таким образом, сформулированный результат показывает, что (при $N=2$ ) ОЛ справедлива на любом открытом п.в. множестве $\Omega \subset T^{2}$ в классе $L\left(\ln ^{+} L\right)^{2}$.

Далее естественно встает вопрос о справедливости ОЛ в “более широком", чем $L\left(\ln ^{+} L\right)^{2}$, классе.

Некоторый ответ на поставленный вопрос дает следующая

ТЕОРема 2. Пусть $\Omega$ - произвольное измеримое множсество $\Omega \subset T^{2}$,

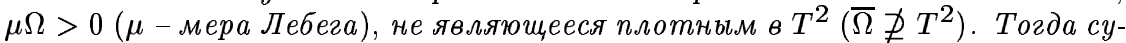
ществуют подмнохество $\Omega_{0} \subset \Omega, \mu \Omega_{0}>0$ и функиия $f \in L\left(\ln ^{+} \ln ^{+} L\right)^{1-\varepsilon}\left(T^{2}\right)$, $0<\varepsilon<1, f(x)=0$ на $\Omega$ такие, что $\varlimsup_{n \rightarrow \infty}\left|S_{n}(x ; f)\right|=+\infty$ для почти вcex $x \in \Omega_{0}$. 
ЗАмечаниЕ. Результат теорем 1 и 2 интересно сравнить с фактом существования функций из класса $L\left(\ln ^{+} L\right)^{1-\varepsilon}\left(T^{2}\right), 0<\varepsilon<1$, с расходящимся п.в. по квадратам двойным рядом Фурье. Этот факт, в частности, следует из работ С. В. Конягина [10] и Р. Д. Гецадзе [11].

5. Пусть функция $g \in L_{p}\left(\mathbb{R}^{N}\right), p \geqslant 1$, разложена в кратный интеграл Фурье:

$$
g(x) \sim \int_{\mathbb{R}^{N}} \widetilde{g}(\xi) e^{i x \xi} d \xi .
$$

Для любого вектора $\alpha=\left(\alpha_{1}, \ldots, \alpha_{N}\right) \in \mathbb{R}_{0}^{N}$ рассмотрим собственный интеграл

$$
J_{\alpha}(x ; g)=\frac{1}{(2 \pi)^{N}} \int_{-\alpha_{1}}^{\alpha_{1}} \cdots \int_{-\alpha_{N}}^{\alpha_{N}} \widetilde{g}(\xi) e^{i\left(x_{1} \xi_{1}+\cdots+x_{N} \xi_{N}\right)} d \xi_{1} \cdots d \xi_{N}
$$

Частньм случаем "прямоугольной частичной суммы” (3) является "квадратная частичная сумма" $J_{\alpha_{0}}(x ; g)$, когда $\alpha_{1}=\alpha_{2}=\cdots=\alpha_{N}=\alpha_{0}$.

Пусть $g(x)=f(x)$ при $x \in T^{N}$. Обозначим через $R_{\alpha}(x ; f)$ следующую разность:

$$
R_{\alpha}(x ; f)=R_{\alpha}(x ; f, g)=S_{[\alpha]}(x ; f)-J_{\alpha}(x ; f),
$$

где $[\alpha]=\left(\left[\alpha_{1}\right], \ldots,\left[\alpha_{N}\right]\right) \in \mathbb{Z}_{0}^{N},\left[\alpha_{j}\right]$ - целая часть $\alpha_{j} \in \mathbb{R}^{1}$. Будем предполагать при этом, что

$$
g(x)=0 \quad \text { вне } T^{N} .
$$

6. При $N=1$ для функций $f \in L_{1}\left(T^{1}\right)$ на любом сегменте, целиком лежащем внутри интервала $(-\pi, \pi)$, разность $R_{\alpha}(x ; f)(4)$ стремится равномерно к нулю при $\alpha \rightarrow \infty$ (при этом условие финитности (5) для функций $g$ несущественно) (см. [12, с. 362-363]). В работе [2] И. Л. Блошанский доказал, что для $N=2$ и $p>1$ $R_{\alpha}(x ; f) \rightarrow 0$ при $\alpha \rightarrow \infty$ (т.е. $\min _{1 \leqslant j \leqslant N} \alpha_{j} \rightarrow \infty$ ) для п.в. $x \in T^{2}$ (условие (5) в данном случае несущественно). В этой же работе была выяснена существенность вида сходимости $R_{\alpha}(x ; f)$ и условий $N=2, p>1$. В работе были построены непрерывные функции: функция $f_{1} \in \mathbb{C}\left(T^{2}\right)$ такая, что $\overline{\lim }_{\alpha \rightarrow \infty}\left|R_{\alpha}\left(0, f_{1}\right)\right|=+\infty$, и функция $f_{2} \in \mathbb{C}\left(T^{N}\right), N>2$, такая, что $\overline{\lim }_{\alpha \rightarrow \infty}\left|R_{\alpha}\left(x ; f_{2}\right)\right|=+\infty$ всюду внутри $T^{N}$. В свою очередь, для $p=1$ приведен пример функции $f_{3} \in L_{1}\left(T^{N}\right)$ такой, что $\varlimsup_{\alpha \rightarrow \infty}\left|R_{\alpha}\left(x ; f_{3}\right)\right|=+\infty$ в каждой точке $x \in T^{N}$.

Далее, в работах [13] и [14] И. Л. Блошанский продолжил исследование вопросов равносходимости разложений в кратный ряд и интеграл Фурье, но уже при суммировании по квадратам. Так в работе [14] были построены две суммируемые функции $f$ и $g: f \in L_{1}\left(T^{N}\right), g \in L_{1}\left(\mathbb{R}^{N}\right), N \geqslant 2$, совпадающие на $T^{N}$, кратный ряд Фурье одной из которых (функции $f$ ) неограниченно расходится п.в. (по некоторым подпоследовательностям), в то время как кратный интеграл другой (функции $g$ ) сходится п.в. (по тем же самым подпоследовательностям).

Сформулированные выше результаты ставят вопрос о равносходимости (рассматриваемых разложений) так же, как и для исследуемой в настоящей работе ОЛ, в классах $\Phi(L)$, т.е. в классах, “лежащих между" $L_{1}$ и $L_{p}, p>1$.

7. В настоящей работе нами получены следующие результаты, касающиеся поведения разности (4) при $N=2$ в указанных классах. 
TEOpEMA 3. Ecлu $f \in L\left(\ln ^{+} L\right)^{2}\left(T^{2}\right)$, mo

$$
\lim _{\alpha \rightarrow \infty} R_{\alpha}(x ; f)=0 \quad \text { для почти всех } \quad x \in T^{2} .
$$

Более того, для любого $\varepsilon>0$ существует постоянная $C_{\varepsilon}>0$ такая, что

$$
\left\|\sup _{\alpha \in \mathbb{R}_{0}^{2}}\left|R_{\alpha}(x ; f)\right|\right\|_{L_{1}\left(T_{\varepsilon}^{2}\right)} \leqslant C_{\varepsilon} \int_{T^{2}}|f(x)|\left(\ln ^{+}|f(x)|\right)^{2} d x+C_{\varepsilon}
$$

əде $T_{\varepsilon}^{2}=\left\{x \in \mathbb{R}^{2}:-\pi+\varepsilon \leqslant x_{j} \leqslant \pi+\varepsilon, j=1,2\right\}$.

Tеорема 4. Существует функиия $f \in L\left(\ln ^{+} \ln ^{+} L\right)^{1-\varepsilon}\left(T^{2}\right), 0<\varepsilon<1$, maкая, что $\varlimsup_{\alpha \rightarrow \infty}\left|R_{\alpha}(x ; f)\right|=+\infty$ для почти всех $x \in T^{2}$.

Московский педагогический университет

Поступило

06.04 .96

Исправленный вариант

22.04 .96

\section{СПИСОК ЦИТИРОВАННОЙ ЛИТЕРАТУРЫ}

1. Блошанский И. Л. // Докл. АН СССР. 1978. Т. 242. №1. С. 11-13. 2. Блошанский И. Л. // Матем. заметки. 1975. Т. 18. №2 . С. 153-168. 3. Carleson L. // Acta Math. 1966. V. 116. P. 135-157. 4. Hunt R. A. // Proc. Conf. Edwardsville, 1967: SIU Press Carbondale, 1968. Р. 235-255. 5. Блошанский И. Л. // Изв. АН СССР. Сер. матем. 1989. Т. 53. № 4. С. 675-707. 6. Bloshanskii I. L. // Anal. Math. 1981. V. 7. № 1. Р. 3-36. 7. Блошанский И. Л. // Автореферат дисс. ... д. ф.-м.н. М.: МИАН, 1991. 8. Sjölin P. // Ark. Mat. 1971. V. 9. № 1. P. 65-90. 9. Fefferman C. // Bull. Amer. Math. Soc. 1971. V. 77. № 2. P. 191-195. 10. Конягин С. В. // Тр. МИАН. 1989. Т. 190. С. 102-116. 11. Гецадзе Р. Д. // Сообщ. АН ГССР. 1986. Т. 122. № 2. С. 269-271. 12. Зигмунд А. Тригонометрические ряды. Т. 2. М.: Мир, 1965. 13. Блошанский И. Л. // Изв. АН СССР. Сер. матем. 1976. Т. 40. № 3. С. 685-705. 14. Блошанский И. Л. // Сиб. матем. ж. 1990. T. 31. №1. C. 39-52. 